\title{
A Service for Robust Decision Downloading in Collaborative Networks
}

\author{
Heiko Thimm $^{1}$ and Karsten Boye Rasmussen ${ }^{2}$ \\ ${ }^{1}$ Pforzheim University, School of Engineering, Tiefenbronner Str. 65 \\ D-75015 Pforzheim, Germany \\ heiko.thimm@hs-pforzheim.de \\ ${ }^{2}$ University of Southern Denmark, Department Marketing and Management, \\ Campusvej 55 DK-5230 Odense, Denmark \\ kbr@sam.sdu.dk
}

\begin{abstract}
Theory and observations about the positive influence of robust decision downloading among the employees within single companies can be related to the context of Collaborative Networks were a moderator makes decisions on behalf of the network. Especially, configuration decisions that generate a Virtual Enterprise arranged from the network members must be downloaded robustly within the network. Otherwise, the network collaboration climate can be damaged since in such decisions the network is separated in members that are included and others that are excluded from participation in the Virtual Enterprise. We present an information technological service that generates and downloads explanations from data about the network and about configuration decisions within the network. Through this service robust decision downloading can be performed effectively and with little time effort by moderators thus contributing to a successful network.
\end{abstract}

Keywords: Collaborative Network, Virtual Enterprise, Collaboration Platform, Network Moderator, Data Analysis, Decision Downloading.

\section{Introduction}

The term decision downloading refers to the communication of a decision to those who have not been involved in the decision-making process [4]. So-called robust decision downloading is considered to be the most appropriate mode of decision downloading. Robust downloaders discuss how and why the decision was made, what alternatives were considered, how it fits in with the organizational mission, and how it impacts the organization and employees. Less effective downloaders discuss some or only few of the above issues. Robust decision downloading has a number of positive implications for individual employees including features such as a stronger support of and commitment to the organization, a higher identity with the organization, and the employee perception that the organization is well managed and headed in the right direction [4]. It is a hypothesis of our research that these findings are to a large extent valid to decision making in Collaborative Networks too. We assume that the members of such networks, i.e. companies of typically smaller and medium size, can be compared to the 
individual employees in the classical decision downloading context. We especially investigate the influence of the decision making practice on the network collaboration climate in Collaborative Networks with a human moderator managing the network. By the engagement of a moderator the more time consuming multi-person decision processes can be substituted by the more agile single-person decision processes [11] in which the moderator makes decisions on behalf of the network. We look at networks were the moderator makes decisions concerning configuration acting as an elected manager of the Collaborative Network. Especially are we considering configuration decisions where companies are selected from the network for building a Virtual Enterprise (VE) for the handling of a business opportunity. We refer to these decisions as $V E$ creation decisions.

Although there is no direct proof of our above described hypothesis available we view robust decision downloading as a major obligation for moderators in parallel with the obligation for managers towards employees. In the long run an inappropriate decision downloading can damage the network collaboration climate. This threat for the network can arise easily in particular from an inappropriate downloading of sensitive decisions. We regard VE creation decisions and other decisions that directly influence the economic situation of the individual network members as sensitive decisions.

We understand that due to the restricted amount of time that is available for moderators to deal with the large spectrum of different moderator tasks there is a strong demand for an effective automation of robust decision downloading in Collaborative Networks. In this article we address this need for automation by proposing a novel information technology based service for robust decision downloading. This service complements an earlier developed decision support service specialized on the configuration of VEs [12]. Both services are based on complex data analyses. The data available for the decision support service together with data about moderator sessions recorded in a machine processible format in a corresponding log is analyzed and decision explanations are generated. These explanations consist of different "views" that together provide the decision background as demanded for robust decision downloading. The views contain quantitative data retrieved from a comprehensive data repository that is specialized for VE creation decisions.

The remainder of the article is organized as follows. In Section two VE creation decisions are analyzed from a decision making point of view. In Section three, we present the conceptual framework of our proposed service for decision downloading. Also a typical application scenario for our services is given and the envisaged benefits are discussed. Section four gives an overview of a corresponding prototype. Related work is discussed in Section five. Section six presents our intended future work and concluding remarks.

\section{VE Creation from a Decision Making Perspective}

In the following sections we investigate processes in Collaborative Networks that are concerned with the forming of VEs. We especially focus on moderated Company Networks where a moderator is in charge of the selection of proper companies for a $\mathrm{VE}$ and related communication measures to inform the network. In our investigation general requirements for corresponding information technology based services are identified that are addressed in a later section. 


\subsection{Partner Selection for VE Creation}

The process of handling an external request for information or offer by a network can be divided in three steps. In step one the request is received from the market by the network. In step two negotiations between the requestor and the network as well as further network-internal processing steps are performed. If a signed order contract between the requestor and the network is achieved a third step is completed. It is a characteristic of Collaborative Networks that in this third step the order is fulfilled as a collaborative task by a subset of the network members that form a Virtual Enterprise (VE). In order to be successful on the market a network is required to find for each request an appropriate VE under consideration of the company's profiles and competences, resource utilization states and other company specific criteria as well as criteria that relate to the network as a whole. Thus, the creation of a VE imposes to the network a complex multi-criteria decision problem [12] were hard and soft selection criteria from the perspective of a single network member but also the perspective of the network as a whole need to be reflected. Not only that the number of selection criteria is increasing with the size of the network, the number and complexity of the offered products, and the number of interdependencies between the network members such as overlapping competences between companies. Their relevance for the VE creation is also changing over time as a result of market changes but also changes with respect to the relations among the network members.

Because of the high degree of complexity of the VE creation task IT based solutions that allow to complete this task in a single person effort have been proposed $[1,2,10]$. The common premise of these approaches is that Collaborative Networks are coordinated and managed by an elected human moderator who as a manager performs the VE creation task on behalf of the entire network by effectively applying the descriptions supplied by the network members.

\subsection{On the Need for Robust Downloading of VE Creation Decisions}

The hypothesis of robust decision downloading will place it in the human nature that employees (or members of Collaborative Networks) will feel uncomfortable if decision downloading does not meet the above mentioned criteria for robust decision downloading. We consider this to be especially valid for decisions that influence the company's viability exemplified in the economic situation affected by such decisions as the VE creation decisions. Each individual VE creation decision is based on a separation of the network members into a group of members that will benefit from the decision - they will be assigned to work on a business and thus attracted by a revenue opportunity - and another group that will not benefit. The group of non-benefiters can be further divided into network members that for more obvious reasons have not become a member of the VE. For example, they might not offer any service or product needed for the fulfillment of the business opportunity. However, the group of nonbenefiters can also consist of companies that offer exactly the services and products needed and that still have not been considered for the VE for some additional reasons. For example, they might have been participating in many previous VEs or their membership in the VE might be prevented due to an exclusion constraint of another more important member needed for the VE. Understanding the reasons for this decision can 
require complicated decision analysis including a projection into a future status of the network as a whole.

It can be expected that robust decision downloading is especially important for this specific subset of non-benefiters in order to obtain their acceptance for the VE creation decision. Since it can be assumed that this subset of non-benefiters is changing from decision to decision over the time many of the network members will make some non-benefiter experience. If the non-benefiters are not supported in overcoming this uncomfortable experience by a robust decision downloading the network collaboration climate can severely suffer in the long run.

\section{A Decision Support and Downloading Service for VE Creation}

Decision Support Service. Figure 1 contains a conceptual view of the proposed decision support service. The usage scheme of this service for the creation of VEs consists of three phases. In the first phase, the request is screened and decomposed into request components. The service supports this task by allowing the moderator to browse through products and services of the companies. The resulting request components determine the kind of products and services needed from the network for the handling of the request. In the second phase, a corresponding search profile for the demanded $\mathrm{VE}$ is specified which states a set of criteria for selecting companies and evaluating possible VE alternatives. In order to allow for convenient specification of a search profile a corresponding interactive template is presented to the moderator. In the third phase, the possible VE alternatives are scored reflecting the fit to the criteria given in the search profile. The scoring result is presented to the moderator in the form of a ranked list of VE alternatives that meet the search profile. The list might be empty if step one has included services and products that are not available and the list might be limited to the top priorities if a large number of alternatives are scored.

Through the use of the proposed support service moderators may achieve what is generally regarded as "informed decision making" [6]. That is, by iterating over the above described phases several times with different versions of search profiles the moderator can obtain deep insights into the decision problem space and explore corresponding VE alternatives. The decision security will increase with more and more iterations until the final decision can be made.

In order to allow for an informed decision making the service makes use of a comprehensive information base that contains the product and service offerings of the members of the company network and also their company profiles, competences, and collaboration preferences. The information base also stores the collaboration history of the company network, i.e. records describing previous VEs including information about the corresponding requests and the resulting collaboration experience. Moreover, the information base contains indicators that describe the economic status of the company network such as cash balance, order backlog, resource utilization, and inventory state.

Decision Downloading Service. By using the decision support service the moderator can conveniently find a proper VE alternative that is in turn brought into being. IT support for the workflow in dynamic virtual enterprises has been studied earlier [7]. The decision downloading service described in the following is targeting another 


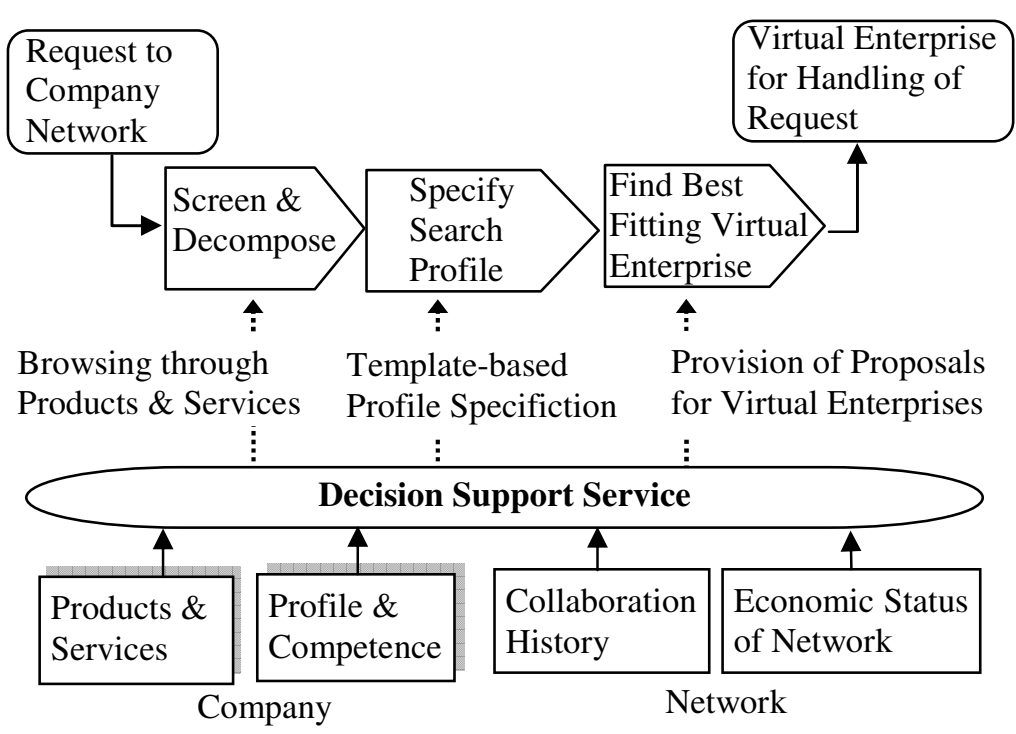

Fig. 1. Proposed Decision Support Service

moderator activity that too has to be performed after it has been decided for a final $\mathrm{VE}$ alternative. It is the goal of this service to perform a robust downloading of the moderator's VE creation decision to the network members where the main target group are the network members that are not selected as part of the VE. For the other network members the decision downloading has a more informative character. Through the downloading service moderators can perform the downloading task efficiently within very limited time without compromising on the richness and userfriendliness of the information that is conveyed. Once the moderator has decided for a particular VE alternative that has been generated by the decision support service the decision downloading service can be instructed to automatically generate and distribute the corresponding explanations within the network. There exist various options for the technical implementation of a distribution mechanism for the explanations such as email newsletters, intranet publishing, and provisioning of explanations in a dedicated online content repository.

The explanations are generated in three steps based on the content of the information base and further data recorded during moderator sessions with the decision support service. In a first step the relevant decision justification information is automatically derived from the individual decision-specific criteria, global policies and strategies defined for the network, and corresponding information about the current global status of the network. In a second step this information is augmented by further context specific background information to make it easier for users to perform a decision diagnosis and to gain understanding about the decision justification. The data are partly contributed by the decision support service that records data for each moderator session including search profiles, intermediate internal processing results, bookkeeping data, and the final ranking of alternative VE creation proposals. In a third step the decision downloading service generates decision explanations in the form of data 
views that are easy to read and understand by humans. By these data views we mean pre-computed views that include quantitative data retrieved from the underlying data collection describing attributes of the network companies.

Views. At the current stage of our research we consider three different types of views. The Search Profile View presents the search profile of the request as decomposed and specified by the moderator. This view gives insights into the needed products and services and the decision criteria that have been considered for the generation of the VE alternatives. The Search Result and Criteria Evaluation View contains the VE alternative for which the moderator has decided finally. This view is intended to clarify to the company representatives the reasons for the final decision and in particular the arguments why their company is a part of the VE or in the opposite case why their company is not part of the VE. The information being presented includes among others the total score and single scores of the chosen VE compared to mean score values of the VE alternatives in terms of the set of relevant decision criteria. The Decision Impact View is based on a projection of quantitative data in the future. Through this view one can learn about future network states from an economic and collaboration point of view as they will result from an order fulfilment by the selected VE. The network states are described in terms of corresponding quantitative indicators such as revenue, resource utilization, inventory state, and collaboration specific indicators. The description is intended to clarify both the consequences of the given VE creation decision for the network as a whole and for the single members of the network. The latter is given in the form of anonymous indicators such as mean number of participations in VEs, mean revenue that companies have obtained as contributor in VEs, mean duration between consecutive VEs that a company is participating in, mean revenue distribution over the network members.

Envisaged Application Scenarios and Benefits. In the following we will describe a fictive example of a small scale company network. The typical application scenarios addressed by our services will share the characteristics of the example except for a bigger size and larger network complexity. We suppose a company network specialized on the production of passenger seats for planes, ships, trains, and busses. Furthermore, we suppose that the moderator needs to select members to form a VE to handle a request for quotation from a shipyard asking for an offer for 400 passenger seats with an integrated infotainment system. From a corresponding process description for the production of the requested seats the moderator can identify or decompose that the following set of activities is needed for the order fulfilment: (1) production of metal seat frames, (2) production of seat upholsteries, (3) production of circuit systems, (4) production of monitors, (5) production of harnesses, (6) final assembly of seats.

The moderator uses the decision support service in order to efficiently explore an appropriate VE - ultimately finding the best fitting VE - among the set of possible alternatives. An initial search profile is accordingly specified which will lead to a list of possible VE configuration alternatives with corresponding scores. The scores reflect the goodness of fit of the VE configuration alternatives with respect to the selection criteria and constraints declared in the search profile. Typically, a moderator will perform several iterations with accordingly modified search profiles until it is finally 
decided for a configuration alternative that has been proposed by the decision support service.

In order to complete the obligation to appropriately download the chosen VE configuration decision the moderator submits the final decision to the downloading service by a corresponding selection operation on the presented result list. Given this activation event the downloading service analyses data recorded during the use of the decision support service and further company and network specific indicators. These analyses lead to decision explanations that are made available as stored documents in a specific repository where they can be accessed by the network members. The members will receive notification emails that contain general data about the decision situation and individualized access information that allows retrieval of the explanation documents.

Through the downloading service moderators can effectively and efficiently perform robust decision downloading. The network members benefit from the service by being supplied with purposefully and consistently prepared decision explanations giving them reliable clarity about reasons for VE creation decisions. Note that without such a service there is the risk that the provisioning as well as the content of the explanations can be biased by human factors. We expect that a decision making practice in Collaborative Networks which is based on the proposed service will contribute to a positive collaboration climate.

\section{Prototype}

A first standalone prototype of our proposed services for Collaborative Networks is currently being implemented. Figure 2 shows the major components of the prototype for which we apply the typical technologies of web-based multi-tier software architectures in combination with the JAVA programming language and other JAVA technologies. The XML standard is used as data interchange format between the system components. For example, session logs, search profiles, search results, and decision explanations are exchanged as XML documents. The prototype offers to moderators a web browser-based front end and another front end for network members specialized on information visualization. The components of the prototype store and maintain data in a common data base which can be logically divided into the four repositories shown in Figure 2. The Company Network Directory (CND) contains descriptions of the companies in terms of their product and service offerings and also their competencies and technical abilities. The VE Creation Log contains recorded sessions in which VE proposals have been generated by the system according to search profiles. The third data repository contains as the name indicates data about the economic status of the network and about collaborative processes and business transactions as completed within the network in reality. Decision explanations are administered in the Decision Views Repository.

The Collaboration Proposal Generator takes the search profile of the moderator and completes a sophisticated orchestration algorithm. A resulting ranked list of VE alternatives is delivered back to the moderator. During such a moderator session the Collaboration Proposal Generator records data about search profiles, processing steps of the orchestration algorithm together with intermediate results, and proposed VE 


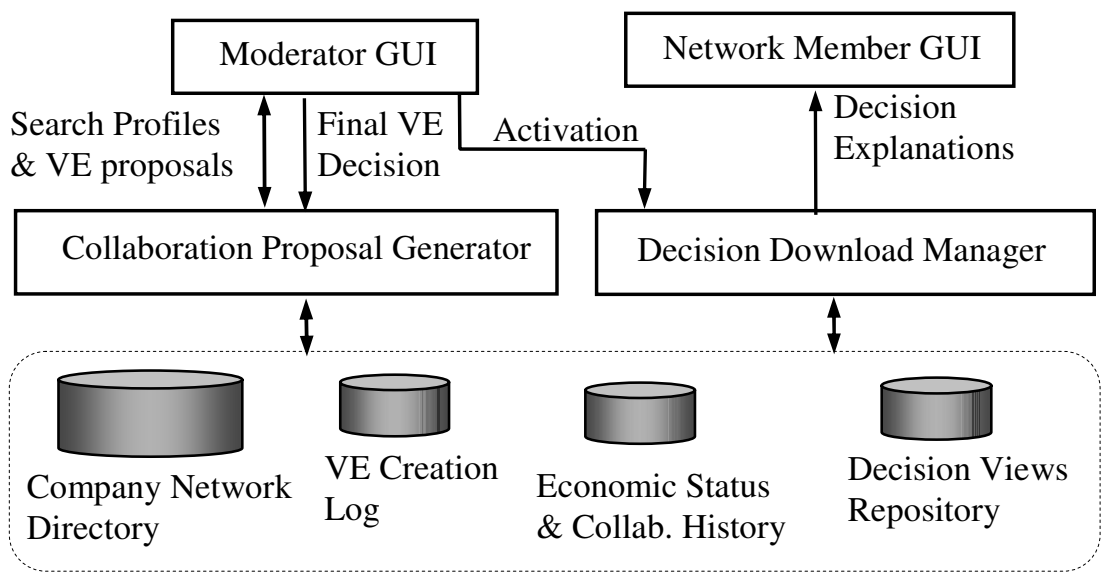

Fig. 2. Architecture of Prototype

alternatives. These data are stored in the VE Creation log for the purpose of automatically generating decision explanations. The Decision Download Manager is called interactively from the moderator GUI when a final decision for a VE alternative is available. The chosen alternative is selected first in the ranked list of alternatives presented to the moderator. This selection is recorded in the VE Creation log and the Decision Download Manager is activated when the moderator has confirmed the selection. The Decision Download Manager then retrieves information from the database to generate the corresponding decision explanations. The generated explanations are made available in the Decision Views Repository where they may be accessed by the network members. In addition to such a direct access, the decision explanations can also be published on the network's intranet. The members are automatically notified by the Download Manager through email messages.

\section{Related Work}

The configuration of Virtual Enterprises from the members of a Collaborative Network has been intensively investigated, for example in the ECOLEAD Project [3]. In many of these studies Virtual Enterprises are the result of a decision or negotiation process in which a multitude of different decision criteria are considered. Because of the complexity of decision criteria it is often difficult to understand the rationales behind such decisions for those uninvolved in the decision making process while the decision makers often believe that the long labored decision needs no explanation [4]. So far not a lot of attention has been paid to the question of how decisions in general and configuration decisions in particular should be communicated in the Collaborative Network. Investigations regarding decision making practices in Collaborative Networks and the impact on the network collaboration climate are only rarely available [9]. This is a surprising fact since decision making and communication in companies as single enterprises and the impact of the outcome and the communication on the individual employee perception of justice or fairness in the company has for long 
been an area of intense research in the concept of "organizational justice" [5, 8]. "Organizational justice" as a perception can be subdivided into or being dependent upon types of justice as "distributive justice", "procedural justice", and "interactional justice" [5]. The distributive concept of justice concerns the fairness of the output given the input. The extend of input from a given company might be more transcendent to all companies as well as to the single company itself due to the use of an information service. Secondly, the "procedural justice" that is covering the fairness in the process will certainly be demonstrated by the proposed service. The software cannot obtain any bias that is not presented as arguments and parameters in the logic of the system. The service will demonstrate how the companies have presented themselves as well as demonstrate the iterative logic in finding the most adequate outcome. The concept of "interactional justice" is about how people are treated with dignity and respect. The procedural aspect mentioned before can be said through the transparent and unbiased system to provide and signify dignity and respect for the individual company. We thus consider these concepts and findings of research on organizational justice as also being relevant for the companies forming Collaborative Networks. We intend to explore this research field in the future for example by simulation studies based on the core models for our moderator services and the prototype.

\section{Future Work and Conclusions}

A great deal of work still lies ahead in elaborating further the introduced decision downloading service. We will especially investigate information visualization concepts [13] with the goal to develop an approach that fits to the specific needs of effective visualization of decision explanations. This will also include solutions allowing the network members to customize the visual presentation of decision explanations to their individual needs. Another more long term goal will be the study of the service's effectiveness with respect to the upkeep of a positive collaboration climate in the Collaborative Network. We intend to experiment with the service on the basis of simulation of the central model. Live tests and first experience with the implementation of the model will necessarily lead to further investigation into the different data views and fact-based indicators that should be contained in decision explanations.

We understand that the network collaboration climate is a crucial success factor of Collaborative Networks and that the decision making practice of the network has a strong influence on this. Without proper IT support it will be extremely difficult for moderators to perform robust decision downloading which is understood as the ideal downloading mode. The article has presented a first approach to such a moderator service for automated robust downloading of VE creation decisions.

\section{References}

1. Bittencourt, F., Rabelo, R.J.: A Systematic Approach for VE Partners Selection Using the Scor Model and the AHP Method. In: Proc. IFIP TC5 Working Conf. on Virtual Enterprises, Valencia, pp. 99-108. Springer, Heidelberg (2005)

2. Camarinha-Matos, L.M.: ICT Infrastructures for VO. In: Camarinha-Matos, L.M., Afsarmanesh, H., Ollus, M. (eds.) Virtual Organisations: Systems and Practices, pp. 83-104. Springer, New York (2005) 
3. Camarinha-Matos, L.M., Afsarmanesh, H.: Collaborative networks: a new scientific discipline. Journal of Intelligent Manufacturing 16, 439-452 (2005)

4. Clampitt, P., Williams, M.: Decision Downloading. MIT Sloan Mgmt. Review (January 2007)

5. Colquitt, J.A., Conlon, D.E., Wesson, M.J., Porter, C.O.L.H., Yee, K., Ng, K.Y.: Justice at the Millennium: A Meta-Analytic Review of 25 Years of Organizational Justice Research. Journal of Applied Psychology 86(3), 425-445 (2001)

6. Dave, G.: Informed decision making. J. of Information Science (51/1), 169-173 (1995)

7. Grefen, P., Aberer, K., Hoffner, Y., Ludwig, H.: CrossFlow: Cross-Organizational Workflow Management in Dynamic Virtual Enterprises. Int. Journ. of Computer Systems Science \& Engineering 15(5), 277-290 (2000)

8. Greenberg, J.: A Taxonomy of Organizational Justice Theories. The Academy of Management Review 12(1), 9-22 (1987)

9. Noran, O.: A Decision Support Framework for Collaborative Networks. In: 8th IFIP Working Conference on Virtual Enterprises (PRO-VE 2007), Guimãraes, Portugal, pp. 8390 (2007)

10. Shen, W., Kremer, R., Ulieru, M., Norrie, D.: A Collaborative Agent-Based Infrastructure for Internet-enabled Collaborative Enterprises. Int. Journal of Production Research 41(8), 1621-1638 (2003)

11. Sherer, S.A.: Critical Success Factors for Manufacturing Networks as Perceived by Network Coordinators. Journal of Small Business Management 41(4), 325-345 (2003)

12. Thimm, H., Thimm, K., Rasmussen, K.: Supporting Moderators of Company Networks by an Optimization Service for Orchestration. In: 16th European Conference on Information Systems (ECIS), Galway, Ireland, June 9-11, p. 12 (2008) ISBN 978-95553159-2-3

13. Zhu, B., Chen, H.: Information Visualization for Decision Making. In: Handbook on Decision Support Systems, vol. 2, pp. 699-772. Springer, Heidelberg (2008) 